\title{
Decreased quality of life and treatment satisfaction in patients with latent autoimmune diabetes of the adult
}

\author{
Minerva Granado-Casas ${ }^{1,2}$, Montserrat Martínez-Alonso ${ }^{3}$, Nuria Alcubierre ${ }^{2}$, Anna Ramírez-Morros ${ }^{1}$, Marta \\ Hernández ${ }^{2,4}$, Esmeralda Castelblanco ${ }^{1}$, Joan Torres-Puiggros ${ }^{5,6}$, Didac Mauricio ${ }^{\text {Corresp. }{ }^{1,2}}$ \\ ${ }^{1}$ Department of Endocrinology and Nutrition, Centre for Biomedical Research on Diabetes and Associated Metabolic Diseases (CIBERDEM), Health \\ Sciences Research Institute \& University Hospital Germans Trias i Pujol, Badalona, Spain \\ 2 Biomedical Research Institute of Lleida, University of Lleida, Lleida, Spain \\ 3 Biostatistics Unit, Biomedical Research Institute of Lleida, University of Lleida, Lleida, Spain \\ 4 Department of Endocrinology and Nutrition, University Hospital Arnau de Vilanova, Lleida, Spain \\ 5 Nursing School, University of Lleida, Lleida, Spain \\ 6 Catalan Department of Health, Lleida, Spain \\ Corresponding Author: Didac Mauricio \\ Email address: didacmauricio@gmail.com
}

Objectives: Our main aim was to assess the quality of life (QoL) and treatment satisfaction (TS) in of subjects with LADA (latent autoimmune diabetes of the adult) and compare these measures with those of patients with other diabetes types, i.e., type 1 (T1DM) and type 2 diabetes mellitus (T2DM).

Methods: This was a cross-sectional study with a total of 48 patients with LADA, 297 patients with T2DM and 124 with T1DM. The Audit of Diabetes-Dependent Quality of Life (ADDQoL-19) questionnaire and the Diabetes Treatment Satisfaction Questionnaire (DTSQ) were administered. Relevant clinical variables were also assessed. The data analysis included comparisons between groups and multivariate linear models.

Results: The LADA patients presented lower diabetes-specific QoL ( $p=0.045)$ and average weighted impact scores $(p=0.007)$ than the T2DM patients. The subgroup of LADA patients with diabetic retinopathy (DR) who were treated with insulin had a lower ADDQoL average weighted impact score than the other diabetic groups. Although the overall measure of TS was not different between the LADA and T2DM ( $p=0.389)$ and T1DM ( $p=0.091)$ groups, the patients with LADA showed a poorer hyperglycemic frequency perception than the T2DM patients $(p<0.001)$ and an improved frequency of hypoglycemic perception compared with the T1DM patients $(p=0.021)$.

Conclusions: The current findings suggest a poorer quality of life, especially in terms of DR and insulin treatment, among patients with LADA compared with those with T1DM and T2DM. Hyperglycemia frequency perception was also poorer in the LADA patients than in the T1DM and T2DM patients. Further research with prospective studies and a large number of patients is necessary. 


\section{Decreased quality of life and treatment satisfaction in patients}

2 with latent autoimmune diabetes of the adult

3 Minerva Granado-Casas ${ }^{1,3}$ RD MSc, Montserrat Martínez-Alonso² PhD, Nuria Alcubierre ${ }^{3}$ RD

4 PhD, Anna Ramírez-Morros ${ }^{1}$ RN MSc, Marta Hernández ${ }^{3,4}$ MD, Esmeralda Castelblanco ${ }^{1}$ PhD,

5 Joan Torres-Puiggros ${ }^{5,6} \mathrm{PhD}$, Dídac Mauricio ${ }^{1,3^{*}} \mathrm{MD}$ PhD

$6{ }^{1}$ Department of Endocrinology and Nutrition, Centre for Biomedical Research on Diabetes and

7 Associated Metabolic Diseases (CIBERDEM), Health Sciences Research Institute \& University

8 Hospital Germans Trias i Pujol, Badalona, Spain

9 'Biostatistics Unit, Biomedical Research Institute of Lleida, University of Lleida, Lleida, Spain

${ }^{3}$ Biomedical Research Institute of Lleida, University of Lleida, Lleida, Spain

${ }^{4}$ Department of Endocrinology and Nutrition, University Hospital Arnau de Vilanova, Lleida,

12 Spain

$13{ }^{5}$ Nursing School, University of Lleida, Spain

${ }^{6}$ Catalan Department of Health, Lleida, Spain

*Corresponding author

Dídac Mauricio MD PhD

University Hospital Germans Trias i Pujol, 08916 Badalona, Spain

Email: didacmauricio@gmail.com 


\section{ABSTRACT}

Objectives: Our main aim was to assess the quality of life (QoL) and treatment satisfaction (TS) in of subjects with LADA (latent autoimmune diabetes of the adult) and compare these measures with those of patients with other diabetes types, i.e., type 1 (T1DM) and type 2 diabetes mellitus (T2DM).

Methods: This was a cross-sectional study with a total of 48 patients with LADA, 297 patients with T2DM and 124 with T1DM. The Audit of Diabetes-Dependent Quality of Life (ADDQoL-19) questionnaire and the Diabetes Treatment Satisfaction Questionnaire (DTSQ) were administered. Relevant clinical variables were also assessed. The data analysis included comparisons between groups and multivariate linear models.

Results: The LADA patients presented lower diabetes-specific QoL ( $p=0.045)$ and average weighted impact scores $(p=0.007)$ than the T2DM patients. The subgroup of LADA patients with diabetic retinopathy (DR) who were treated with insulin had a lower ADDQoL average weighted impact score than the other diabetic groups. Although the overall measure of TS was not different between the LADA and T2DM ( $p=0.389)$ and T1DM ( $p=0.091)$ groups, the patients with LADA showed a poorer hyperglycemic frequency perception than the T2DM patients $(p<0.001)$ and an improved frequency of hypoglycemic perception compared with the T1DM patients $(p=0.021)$

Conclusions: The current findings suggest a poorer quality of life, especially in terms of DR and insulin treatment, among patients with LADA compared with those with T1DM and T2DM. Hyperglycemia frequency perception was also poorer in the LADA patients than in the T1DM 
41 and T2DM patients. Further research with prospective studies and a large number of patients is

42 necessary. 
43

44

\section{INTRODUCTION}

Latent autoimmune diabetes in adults (LADA) is a slowly progressive form of autoimmune diabetes that presents an initial type 2 diabetes mellitus (T2DM) phenotype combined with diabetes-related autoantibodies (Leslie, Palmer, Schloot \& Lernmark, 2016). At diagnosis, patients do not require insulin therapy and are often classified as T2DM patients (Hawa et al., 2013; Stenstrom, Gottsater, Bakhtadze, Berger, \& Sundkvist, 2005). Patients with LADA are younger and leaner that T2DM patients (Hawa et al., 2013; Stenstrom et al., 2005). They usually have a lower body mass index (BMI), serum triglycerides (TG), waist circumference (WC), waistto-hip ratio, and blood pressure (BP) and higher HDL cholesterol levels than the T2DM population (Fourlanos et al., 2005; Hawa et al., 2009, 2013; Isomaa et al., 1999; Mollo et al., 2013). The cardiovascular risk profile of LADA is intermediate, falling between that of type 1 and type 2 diabetes. Additionally, these patients show lower residual endogenous insulin secretion and progress more rapidly to insulin treatment with worse glycemic control (Fourlanos et al., 2005; Hawa et al., 2009; Isomaa et al., 1999; Mollo et al., 2013).

Quality of life (QoL) is a subjective measure of health and well-being related to disease. It includes psychosocial features, physical functioning, mobility and personal care (Esteban y Peña et al., 2010). The aim of measuring QoL is to provide a more comprehensive, integral, precise and valid evaluation of patients' health status (Speight, Reaney, \& Barnard, 2009). Treatment satisfaction (TS) is an individual subjective measure that assesses the patients' experience of treatment processes and results, including ease of use, side effects and efficacy (Hervás A, Zabaleta A, de Miguel G, Beldarrain O, 2002). TS can be influenced by demographic characteristics such as age, educational level and salary (Villar-López J, Lizán-Tudela L, Soto- 
65 Alvarez J, 2009). The development of the disease, information regarding treatment, therapy

66

67

68

69 availability and costs are also associated with TS (Villar-López J, Lizán-Tudela L, Soto-Alvarez J, 2009). Furthermore, diabetes mellitus reportedly has a negative impact QoL, but TS is optimal in this population (Speight et al., 2009).

Previous studies have investigated the QoL and TS of patients with type 1 diabetes mellitus (T1DM) and T2DM, but not those with LADA (Ahola et al., 2010; Bradley et al., 2011; DepablosVelasco et al., 2014; Nicolucci et al., 2009; Oliva, Fernandez-Bolanos, \& Hidalgo, 2012). The PANORAMA study found that T2DM patients with poor glycemic control, complex treatments and severe hypoglycemic episodes showed poorer QoL compared with patients without these factors (Bradley et al., 2011; Depablos-Velasco et al., 2014). Other studies observed that QoL and treatment satisfaction were lower with increasing age, female sex, lower education level, insulin treatment and obesity, the presence of diabetic comorbidities, poorer glycemic control and lower socioeconomic status (Ahola et al., 2010; Nicolucci et al., 2009; Oliva et al., 2012).

As the diagnosis of LADA among subjects with type 2 diabetes is usually delayed, which may increase the disease burden, we hypothesized that LADA patients might have a lower QoL and TS than their counterparts with T2DM; however, LADA is an autoimmune form of diabetes that usually evolves into a phenotype closely related to that of classical type 1 diabetes patients. Furthermore, the identification of LADA subjects may be relevant from the clinical point of view.

To our knowledge, no studies have evaluated QoL and TS in subjects with LADA. Therefore, the primary objective of this study was to assess QoL and TS in patients with LADA and to compare 
86 these measures with those of T2DM and T1DM patients. We also evaluated the factors related

87 to both QoL and TS in the study subjects.

88

MATERIALS AND METHODS

90

91

92

93

94

95

96

97

The study design was observational and cross-sectional. LADA was defined as diabetes diagnosed in individuals over 30 years of age with a positive test for glutamic acid decarboxylase (GAD) antibodies and without the need for insulin treatment during the first 6 months after diagnosis (Leslie et al., 2008; Mollo et al., 2013). Patients with T1DM and T2DM were diagnosed according to the current standard diagnostic criteria, as described previously (Mollo et al., 2013). The inclusion criteria for the three groups of patients were as follows: a diagnosis of diabetes mellitus with a disease duration of more than one year; age greater than 18 years; absence of disability due to macrovascular complications (including a history of diabetic foot disease); and macroalbuminuria (urine albumin-to-creatinine ratio $<300 \mathrm{mg} / \mathrm{g}$ ) or renal failure (estimated glomerular filtration rate $>60 \mathrm{ml} / \mathrm{min} / 1.73 \mathrm{~m}^{2}$ ). The exclusion criteria were as follows: conditions that could affect the results, such as dementia, mental diseases, hearing and languages problems, pregnancy and disability due to cardiovascular diseases.

Dementia and mental diseases had to be diagnosed by a physician and could be determined using the registered clinical records of the patient's general practitioner. No screening tools for detecting initial cognitive impairment were used. All included patients had an estimated glomerular filtration rate $>60 \mathrm{ml} / \mathrm{min}$, except for two LADA participants who had a glomerular filtration rate between 30 and $59 \mathrm{ml} / \mathrm{min}$. Two patients in the T1DM group had previous cerebrovascular events, and one patient had bilateral femoral stenosis; none of the patients 
108 had any disability. The study was approved by the Ethics Committee of Hospital Arnau de

109 Vilanova (Ethical Application Ref: CEIC 1079 and Ref: PI-13-095). Written informed consent was

110 obtained from all of the subjects.

111

\section{Clinical variables}

113 Trained researchers (M.G-C and NA) conducted personal interviews with each of the patients

114 and reviewed the medical records to collect the data regarding the variables of interest.

115 Anthropometric measures were determined according to standard criteria. Laboratory tests

116 and blood pressure were measured using standard procedures as previously described

117 (Alcubierre et al., 2014). Hypertension and dyslipidemia were defined if the participant was

118 undergoing pharmacological treatment for these conditions. Microalbuminuria was defined as

119 an albumin creatinine ratio $>30 \mathrm{mg} / \mathrm{g}$. Physical activity was assessed using a validated method

120 by Bernstein, Morabia, \& Sloutskis (1999); participants were classified as engaging in regular

121 exercise if they performed any physical activity that required at least $\geq 4$ METS (The Metabolic

122 Equivalent) of brisk walking for 30 minutes or more and as sedentary if they did not perform

123 any daily physical activity or if they engaged less than 30 minutes of physical activity per day

124 (Bernstein et al., 1999; Cabrera de León et al., 2007).

125

\section{Quality of life}

127 QoL was assessed through the Audit of Diabetes-Dependent Quality of Life (ADDQoL-19), a

128 disease-specific QoL questionnaire designed and validated in diabetic Spanish subjects (Bradley 
129

130

131

132

133

134

135

136

137

138

139

140

141

142

143

144

145

146

147

148

149

et al., 2011; Bradley C, Todd C, Gorton T, Symonds E, Martin A, 1999; Depablos-Velasco et al., 2014) (Supplementary File 1). This questionnaire consists of 21 items, of which 19 are related to specific life domains and are scored on a 5-point scale. The impact of diabetes on each domain is weighted according to the importance of the domain to the patient's QoL and is reported as the average weighted impact score. These scores can range from +9 (maximum positive impact) to -3 (maximum negative impact). The first two items are general and are scored separately. The first item measures current QoL and is scored from +3 (excellent) to -3 (very bad). The second item measures diabetes-specific QoL and ranges from -3 (maximum negative impact) to +1 (maximum positive impact). Moreover, five of the 19 items that may not have importance for some patients are included in a preliminary question that can be ignored if not applicable. All the questionnaires were administered individually by two trained interviewers (M.G-C and N.A.).

\section{Treatment satisfaction}

TS was determined by a diabetes-specific questionnaire, the Diabetes Treatment Satisfaction Questionnaire status-version (DTSQ-s) (C Bradley, 1994), that has been validated for the Spanish diabetic population (Gomis, Herrera-Pombo, Calderón, Rubio-Terrés, \& Sarasa, 2006) (Supplementary File 2). This questionnaire consists of 8 items scored on a 6-point scale. The final score is weighted according to 6 items with total scores ranging from 36 (very satisfied) to 0 (very unsatisfied). The two remaining items measure the frequency of hyperglycemia and hypoglycemia, respectively, and are scored on a scale from 0 (never) to 6 (always). 


\section{Sample size}

151 To our knowledge, no previous studies have reported QoL and TS in patients with LADA.

152 Additionally, the number of subjects with LADA is limited at the local level, and we aimed to 153 recruit all available patients.

\section{Statistical analysis}

The statistical analysis included the comparability among the groups of subjects with diabetes (LADA, T2DM and T1DM) and multivariate linear regression models' estimation of the variability of the overall mean score for QoL and the present QoL and diabetes-specific QoL items provided by the ADDQoL questionnaire. The TS score and the hyperglycemia and hypoglycemia frequencies were also fitted in multivariate linear regression models to identify differences between the types of diabetes after adjustment for significantly related patient characteristics. The comparison of QoL and TS between the diabetic groups was stratified by the median of the two groups. In the multivariate linear regression models, LADA patients undergoing insulin treatment were used as the reference group in all of the analyses. The comparability analysis included Pearson's chi-squared test (or Fisher's exact test in the case of any expected frequency lower than 5) to compare the distribution of qualitative characteristics. The Kruskal-Wallis test was performed to compare the distribution of quantitative characteristics, including pairwise comparisons to adjust for multiple testing according to the Benjamini \& Hochberg method; furthermore, these characteristics were described by median and interquartile intervals for 
170

171

172

173

174

175

176

177

178

179

180

181

182

183

184

185

each diabetic group. The significance level was set at 0.05 . The statistical software $R$ version

3.3.2 was used for the analyses.

\section{RESULTS}

We had previously identified a total of 106 LADA patients in the local cohort of the only reference hospital in Lleida (North-Eastern Spain). This hospital is the public reference center for specialized diabetes care for the health care district of Lleida. From this sample, 20 participants were excluded after the initial screening based on the exclusion criteria. From a sample of 86 LADA patients who were contacted to participate in the study, 51 participants initially accepted, and an additional 3 patients were excluded. Thus, 48 LADA patients were included in the study. From a sample of 170 T1DM patients who were contacted to participate in the study, a total of 127 agreed to participate, and 3 were excluded for pregnancy, yielding a final sample of 124 patients. As a comparison with T2DM, we used the entire group of 297 patients with T2DM who had been included in a previous study of QoL conducted at the same center (Alcubierre et al., 2014).

The comparison between the patients with LADA included in the study and those who refused to participate $(n=35)$ revealed no differences in clinical characteristics except for a lower frequency of insulin treatment in the non-participant group (72\%; $p=0.049$ for the comparison with the LADA patients included in the current study).

The clinical and demographic characteristics of the study groups are shown in Table 1 . The LADA patients had an intermediate cardiovascular risk profile in terms of adiposity, lipids and 
191 blood pressure compared with the T2DM and T1DM groups. The patients with LADA were older

192 than those with T1DM ( $p<0.001)$. The duration of diabetes in the LADA group was longer $(10.7$

193 years) than that of the T2DM group (8 years) and shorter than that of the T1DM group (20.5

194 years; $p<0.001)$. The frequency of diabetic retinopathy (DR) was $22.9 \%$ in the LADA group,

$19549.8 \%$ in the T2DM group and $40.3 \%$ in the T1DM group ( $p=0.001)$. There were no differences

196 in glycated hemoglobin (HbA1c) among the 3 groups $(p=0.689)$.

197 Quality of life

198 The scores for current QoL did not differ among the 3 study groups ( $p=0.503$; Table 2);

199 however, there was a higher proportion of patients with negative diabetes-specific QoL in the

200 LADA group (70.8\%) than in the T2DM group (52.9\%; $p=0.045)$. Furthermore, more subjects

201 with LADA had a negative average weighted impact score (60.4\%) compared with the T2DM

202 patients (37.7\%; $p=0.007)$. Concerning these QoL measures, we could not find differences

203 between the LADA and T1DM patients. 
Table 1. Clinical characteristics of the study groups

\begin{tabular}{|c|c|c|c|c|c|c|}
\hline Characteristics & LADA $(\mathrm{N}=48)$ & T2DM (N=297) & T1DM (N=124) & $p$-value* & p-LADA vs. T2DM & p-LADA vs. T1DM \\
\hline Age (years) & $62[53.8 ; 70.2]$ & $60[52.0 ; 68.0]$ & $46[37.0 ; 53.0]$ & $<0.001$ & 0.118 & $<0.001$ \\
\hline Sex, male & $26(54.2)$ & $151(50.8)$ & $57(46.0)$ & 0.543 & & \\
\hline Education level & & & & $<0.001$ & 0.181 & 0.012 \\
\hline$<$ Primary & $3(6.3)$ & $38(12.8)$ & $17(13.7)$ & & & \\
\hline Primary & $24(50.0)$ & $169(56.9)$ & $31(25.0)$ & & & \\
\hline Secondary & $18(37.5)$ & $69(23.2)$ & $54(43.5)$ & & & \\
\hline Graduate or higher & $3(6.3)$ & $21(7.1)$ & $22(17.7)$ & & & \\
\hline Smoking & & & & 0.891 & & \\
\hline Non-smoker & $24(50.0)$ & $139(47.3)$ & $57(46.0)$ & & & \\
\hline Smoker, current & $11(22.9)$ & $62(21.1)$ & $31(25.0)$ & & & \\
\hline Smoker, former & $13(27.1)$ & 93 (31.6) & $36(29.0)$ & & & \\
\hline Physical activity & & & & 0.052 & & \\
\hline Sedentary & $13(27.1)$ & $114(38.4)$ & $34(27.4)$ & & & \\
\hline Regular physical activity & 35 (72.9) & $183(61.6)$ & $90(72.6)$ & & & \\
\hline Diabetes duration (years) & $10.7[6.5 ; 16.7]$ & $8[4.0 ; 15.0]$ & $20.5[14.0 ; 30.2]$ & $<0.001$ & 0.035 & $<0.001$ \\
\hline $\mathrm{BMI}\left(\mathrm{kg} / \mathrm{m}^{2}\right)$ & $27.1[24.1 ; 30.4]$ & $30.6[28.1 ; 34.7]$ & $24.6[22.5 ; 27.2]$ & $<0.001$ & $<0.001$ & 0.001 \\
\hline Waist (centimeters) & $96.7 \pm 15.5$ & $106 \pm 11.8$ & $87.7 \pm 12.8$ & $<0.001$ & $<0.001$ & $<0.001$ \\
\hline Hypertension & $26(54.2)$ & $168(56.6)$ & $41(33.1)$ & $<0.001$ & 0.878 & 0.027 \\
\hline Systolic blood pressure (mmHg) & $134[124.0 ; 148.0]$ & $139[127.0 ; 150.0]$ & $127[113.0 ; 139.0]$ & $<0.001$ & 0.220 & 0.017 \\
\hline Diastolic blood pressure (mmHg) & $73[69.0 ; 79.0]$ & $77[70.0 ; 84.0]$ & $73[65.0 ; 78.2]$ & $<0.001$ & 0.126 & 0.235 \\
\hline Dyslipidemia & $34(70.8)$ & $131(44.1)$ & $55(44.4)$ & 0.002 & 0.003 & 0.005 \\
\hline Diabetic retinopathy & $11(22.9)$ & $148(49.8)$ & $50(40.3)$ & 0.001 & 0.003 & 0.075 \\
\hline Microalbuminuria & $11(23.4)$ & $43(14.5)$ & $11(9.1)$ & 0.051 & & \\
\hline Insulin treatment & $43(89.6)$ & $97(32.7)$ & $124(100.0)$ & $<0.001$ & $<0.001$ & 0.001 \\
\hline HbA1c (\%) & $7.5[6.9 ; 8.2]$ & $7.6[6.8 ; 8.5]$ & $7.6[7.0 ; 8.1]$ & 0.689 & & \\
\hline Total cholesterol (mg/dL) & $176[155.0 ; 202.0]$ & $181[163.0 ; 205.0]$ & $182[165.0 ; 202.0]$ & 0.553 & & \\
\hline $\mathrm{HDL}-\mathrm{c}(\mathrm{mg} / \mathrm{dL})$ & $58.5[40.8 ; 70.2]$ & $48[41.8 ; 59.0]$ & $63[53.9 ; 74.0]$ & $<0.001$ & 0.040 & 0.011 \\
\hline LDL-c (mg/dL) & $101[84.0 ; 123.0]$ & $106[87.2 ; 128.0]$ & $102[89.6 ; 116.0]$ & 0.391 & & \\
\hline Triglycerides (mg/dL) & $91.5[66.8 ; 134.0]$ & 117 [83.0;167.0] & $65.5[53.8 ; 81.2]$ & $<0.001$ & 0.003 & $<0.001$ \\
\hline
\end{tabular}

*p-value for comparison between groups. LADA: latent autoimmune diabetes of adult, T2DM: type 2 diabetes mellitus, T1DM: type 1 diabetes mellitus, BMI: body mass index, HbA1c: glycated haemoglobin, HDL-c: high-density lipoprotein cholesterol, LDL-c: low-density lipoprotein cholesterol. Data are median [interquartile], $\mathrm{n}(\%)$ or means \pm SD. 
204 The multivariate linear model of the diabetes-specific QoL score revealed a significant

205 interaction with insulin treatment, as indicated in Table 3. The results are expressed using the

206 group of insulin-treated patients with LADA as the reference group. The LADA and T2DM

207 subjects without insulin treatment showed a higher diabetes-specific QoL than the reference

208 group ( $p=0.004$ and $p<0.001$, respectively), whereas there were no differences between the

209 patients with T1DM and insulin-treated T2DM patients. Additionally, the presence of

210 hypertension, longer disease duration and a larger waist circumference had a negative impact

211 on diabetes-specific QoL.

Table 2.Descriptive analysis for the Audit of Diabetes Dependent Quality of Life (ADDQoL) results of the study groups

\begin{tabular}{|c|c|c|c|c|c|c|}
\hline Items & $\begin{array}{c}\text { LADA } \\
(\mathrm{N}=48) \\
\mathrm{n}(\%)\end{array}$ & $\begin{array}{c}\text { T2DM } \\
(\mathrm{N}=297) \\
\mathrm{n}(\%)\end{array}$ & $\begin{array}{c}\text { T1DM } \\
(\mathrm{N}=124) \\
\mathrm{n}(\%)\end{array}$ & $p$-value* & $\begin{array}{c}p \text {-LADA } \\
\text { vs. T2DM }\end{array}$ & $\begin{array}{l}\text { p-LADA } \\
\text { vs. T1DM }\end{array}$ \\
\hline Present QoL & & & & 0.503 & & \\
\hline$[-3,2)$ & $42(87.5)$ & $242(81.5)$ & $99(79.8)$ & & & \\
\hline$[2,3]$ & $6(12.5)$ & $55(18.5)$ & $25(20.2)$ & & & \\
\hline Diabetes-specific QoL & & & & $<0.001$ & 0.045 & 0.634 \\
\hline$[-3,0)$ & $34(70.8)$ & $157(52.9)$ & $94(75.8)$ & & & \\
\hline$[0,2]$ & $14(29.2)$ & $140(47.1)$ & $30(24.2)$ & & & \\
\hline Average weighted impact score & & & & $<0.001$ & 0.007 & 0.069 \\
\hline$[-6.526,-0.842)$ & $29(60.4)$ & $112(37.7)$ & $94(75.8)$ & & & \\
\hline$[-0.842,0.316]$ & 19 (39.6) & $185(62.3)$ & $30(24.2)$ & & & \\
\hline
\end{tabular}

The groups are stratified by medians.

*p-value for comparison between groups.

QoL: quality of life, LADA: latent autoimmune diabetes of adult, T2DM: type 2 diabetes mellitus, T1DM: type 1 diabetes mellitus

212 Concerning the ADDQoL average weighted impact score, we found a significant interaction

213 between DR, the study group (i.e., type of diabetes) and insulin treatment (Table 4). Insulin-

214 treated LADA subjects showed a poorer average weighted impact score than their

215 corresponding type 2 non-insulin-treated counterparts ( $p<0.001)$. Additionally, T2DM patients

216 with DR and with $(p=0.01)$ or without $(p=0.03)$ concomitant insulin treatment and T1DM

217 patients with DR ( $p=0.03)$ had a better average weighted impact score than LADA patients 
218 undergoing insulin treatment. The presence of DR, longer disease duration, lower education

219 level (less than a primary education) and former smoking had a negative impact on the average

220 weighted impact score. Nevertheless, physical activity was positively related to this measure of

221 QoL ( $p=0.010)$. Furthermore, using the LADA patients undergoing insulin treatment with or

222 without DR as reference groups, we could estimate the combined coefficients using the same

223 model (Table S1). These analyses showed that LADA subjects with DR who were treated with

224 insulin showed a lower QoL than any other combination of diabetes type, insulin treatment and

225 DR. Furthermore, the LADA patients undergoing insulin treatment without DR had a lower QoL

226 than the T2DM patients without insulin treatment either with $(p=0.006)$ or without DR

$227(p<0.001)$

Table 3.Multivariate linear regression for the Audit of Diabetes Dependent Quality of Life (ADDQoL) diabetes-specific QoL score

\begin{tabular}{lccc}
\hline Coefficients & Estimate & Standard error & $p$ value \\
\hline Intercept & 0.278998 & 0.407968 & 0.490 \\
T2DM * without insulin & 0.815249 & 0.154823 & $<0.001$ \\
LADA * without insulin & 1.187429 & 0.415424 & 0.004 \\
T2DM * insulin & 0.238438 & 0.168811 & 0.160 \\
T1DM & 0.024457 & 0.164031 & 0.880 \\
Hypertension & -0.310550 & 0.089091 & 0.001 \\
HbA1c & -0.070135 & 0.035407 & 0.050 \\
Disease duration & -0.010606 & 0.004989 & 0.030 \\
Waist circumference & -0.008147 & 0.003458 & 0.020 \\
\hline
\end{tabular}

Multiple R-squared: 0.2284 ( 27 cases with missing information for any variable in the model).

Reference group: LADA patients receiving insulin treatment.

*Indicates the existence of interactions between variables.

LADA: latent autoimmune diabetes of adult, T2DM: type 2 diabetes mellitus, T1DM: type 1 diabetes mellitus, HbA1c: glycated haemoglobin. 
229 The proportion of subjects with a lower DTSQ final score differed among the study groups:

230 LADA, 60.4\%; T2DM, 52.5\%; and T1DM, 41.9\% ( $p=0.049)$. However, individual paired

231 comparisons between the groups did not yield statistically significant differences (Table 5). The

232 multivariate linear regression analysis of the DTSQ final score revealed no differences between

233 the different combinations of groups according to diabetes type and insulin-treatment (Table

234 S2). Physical activity had a positive impact $(p=0.001)$ and former smoking had a negative impact

235 on the DTSQ final score $(p=0.01)$.

Table 4.Multivariate linear regression for the Audit of Diabetes Dependent Quality of Life (ADDQoL) average weighted impact score

\begin{tabular}{lccc}
\hline Coefficients & Estimate & Standard error & p value \\
\hline Intercept & -1.29375 & 0.21782 & $<0.001$ \\
T2DM * without insulin & 0.95200 & 0.20699 & $<0.001$ \\
LADA * without insulin & 1.06176 & 0.64037 & 0.100 \\
T2DM * insulin & 0.10006 & 0.31884 & 0.750 \\
T1DM & -0.04103 & 0.22328 & 0.850 \\
DR & -1.25952 & 0.40942 & 0.002 \\
Disease duration & -0.01674 & 0.00663 & 0.010 \\
No education* & -0.45008 & 0.15383 & 0.004 \\
Physical activity & 0.27361 & 0.10539 & 0.010 \\
Smoker, current & -0.21031 & 0.12937 & 0.100 \\
Smoker, former & -0.30882 & 0.11674 & 0.008 \\
T2DM * without insulin * DR & 0.92912 & 0.43927 & 0.030 \\
LADA * without insulin * DR & 1.10620 & 1.05301 & 0.290 \\
T2DM * insulin * DR & 1.23910 & 0.49206 & 0.010 \\
T1DM * DR & 0.98320 & 0.44443 & 0.030 \\
\hline
\end{tabular}

Multiple R-squared: 0.2895 ( 6 cases with missing information for any variable in the model).

Reference group: LADA patients receiving insulin treatment.

*Indicates the existence of interactions between variables.

* "No education” identifies patients who did not complete compulsory education.

LADA: latent autoimmune diabetes of adult, T2DM: type 2 diabetes mellitus, T1DM: type 1 diabetes mellitus, DR: diabetic retinopathy 
236 Concerning another measure of TS, the proportion of patients with a perception of increased

237 hyperglycemia frequency perception was higher in the LADA group (87.5\%) than in the T2DM

238 (53.9\%) and T1DM groups (71\%; $p<0.001$ and $p=0.039$, respectively). The multivariate linear

239 regression analysis found an interaction between the type of diabetes and insulin treatment

240 (Table S3). The insulin-treated LADA patients had a higher hyperglycemia frequency perception

241 than the T1DM $(p=0.04)$ and insulin-treated T2DM subjects $(p=0.05)$. Physical activity was

242 associated with a lower hyperglycemia perception frequency ( $p=0.002)$. Additionally, $D R$ and

243 higher $\mathrm{HbA1c}$ were associated with higher hyperglycemia frequency perception ( $\mathrm{p}=0.05$ and

$244 p<0.001$, respectively).

Table 5. Results of the variables corresponding to the Diabetes Treatment Satisfaction Questionnaire (DTSQ) in the different study groups

\begin{tabular}{|c|c|c|c|c|c|c|}
\hline Items & $\begin{array}{c}\text { LADA } \\
(\mathrm{N}=48) \\
\mathrm{n}(\%)\end{array}$ & $\begin{array}{c}\text { T2DM } \\
(\mathrm{N}=297) \\
\mathrm{n}(\%)\end{array}$ & $\begin{array}{c}\text { T1DM } \\
(\mathrm{N}=124) \\
\mathrm{n}(\%)\end{array}$ & p-value* & $\begin{array}{l}\text { p-LADA } \\
\text { vs. T2DM }\end{array}$ & $\begin{array}{l}\text { p-LADA } \\
\text { vs. T1DN }\end{array}$ \\
\hline $\begin{array}{l}\text { Hyperglycemia } \\
\text { frequency perception }\end{array}$ & & & & $<0.001$ & $<0.001$ & 0.039 \\
\hline $0-2$ & $6(12.5)$ & $137(46.1)$ & $36(29)$ & & & \\
\hline $3-6$ & $42(87.5)$ & 160 (53.9) & $88(71)$ & & & \\
\hline $\begin{array}{l}\text { Hypoglycemia } \\
\text { frequency perception }\end{array}$ & & & & $<0.001$ & 0.095 & 0.021 \\
\hline $0-2$ & $32(66.7)$ & $234(78.8)$ & $55(44.4)$ & & & \\
\hline $3-6$ & $16(33.3)$ & $63(21.2)$ & $69(55.6)$ & & & \\
\hline Final score & & & & 0.049 & 0.389 & 0.091 \\
\hline$[4,28)$ & $29(60.4)$ & $156(52.5)$ & 52 (41.9) & & & \\
\hline$[28,36)$ & 19 (39.6) & 141 (47.5) & $72(58.1)$ & & & \\
\hline
\end{tabular}

The groups are stratified by medians.

*p-value for comparisons between groups.

LADA: latent autoimmune diabetes of adult, T2DM: type 2 diabetes mellitus, T1DM: type 1 diabetes mellitus

245 Finally, the proportion of patients with higher hypoglycemia frequency perception differed

246 among the groups ( $p<0.001$; Table 5). A higher proportion of patients with T1DM (55.6\%) than

247 patients with LADA (33.3\%) reported a high frequency of perceived hypoglycemia $(p=0.021)$. In

248 the multivariate linear regression analysis (Table S4), T2DM patients without insulin treatment 
249 showed a significantly lower hypoglycemia frequency perception compared with insulin-treated

250 LADA subjects $(p=0.006)$. Additionally, the following conditions increased this measure: T1DM

$251(p=0.02)$, female sex $(p=0.001)$ and disease duration $(p=0.006)$.

252

253

\section{DISCUSSION}

254 In the current study, we demonstrated that LADA patients presented with lower diabetes-

255 associated specific and average weighted impact QoL scores than patients with T2DM;

256 however, we found no differences in terms of present QoL. Insulin treatment had a negative

257 impact on diabetes-specific QoL, and the subgroup of insulin-treated patients with LADA did not

258 differ from other insulin-treated groups (T2DM and T1DM) in this regard; however, the average

259 weighted impact score was poorer in insulin-treated LADA subjects than in their corresponding

260 T2DM counterparts. The group with the worst values for this impact score was the subgroup of

261 LADA patients with DR and insulin treatment.

262 Although there was a significant difference in the DTSQ final score among the groups, paired comparisons between groups did not reach significant differences; however, there were differences concerning the hyperglycemia and hypoglycemia frequency perception among the groups. The LADA patients showed an increased hyperglycemia frequency perception compared with the T2DM and T1DM groups, which was mainly at the expense of the insulintreated LADA group. In contrast, the LADA patients had an improved hypoglycemia perception

268 frequency compared with the T1DM patients.

269 To our knowledge, there are no previous studies on QoL and TS in subjects with LADA.

270 Concerning quality of life, previous studies revealed a lower QoL in patients with type 1 
271 diabetes or insulin-treated type 2 diabetes and in subjects with one or more late diabetic

272 complications (Collins, O'Sullivan, Harkins, \& Perry, 2009). In a previous study by our group, we

273 also found that patients with T2DM and DR had a lower QoL than those without this

274 complication (Alcubierre et al., 2014), which is in line with the current findings in patients with

275 LADA. Our results are also in line with other previous studies that reported that insulin therapy

276 and diabetic complications were associated with a poorer QoL in patients with T2DM (Bradley

277 \& Speight, 2002; Collins et al., 2009; Depablos-Velasco et al., 2014; Shim, Lee, Toh, Tang, \& Ko,

278 2012; Speight \& Bradley, 2000; Sundaram et al., 2007); however, in a longitudinal study of

279 patients with T2DM starting insulin therapy, QoL improved six months after the

280 commencement of insulin therapy (Wilson, Moore, \& Lunt, 2004). The negative impact of

281 insulin treatment on LADA patients is associated with a poorer QoL perception, which may be

282 linked impaired metabolic control and the delayed initiation of insulin treatment. Furthermore,

283 DePablos-Velasco et al. (2014) observed that diabetic patients with poor metabolic control had

284 a low QoL; however, we found no significant differences in terms of glycemic control.

285 Shim et al. (2012) described that the ADDQoL average weighted impact score was lower in

286 association with male gender, higher education level and longer disease duration. Other studies

287 have related lower QoL to advanced age, female sex, lower educational level, obesity, the

288 presence of diabetic comorbidities, poorer glycemic control and lower socioeconomic status in

289 the type 1 and type 2 diabetic populations (Ahola et al., 2010; Bradley \& Speight., 2002; Collins

290 et al., 2009; Imayama, Plotnikoff, Courneya \& Johnson, 2011; Nicolucci et al., 2009; Oliva et al.,

291 2012; Shim et al., 2012; Speight \& Bradley, 2000; Sundaram et al., 2007). These findings are 
292 similar to our results concerning the presence of complications (i.e., DR), longer disease

293 duration and waist circumference, all of which were related to a lower QoL.

294 However, we found a positive relationship between QoL and physical activity in patients with

295 diabetes. Physical activity is an important component of the lifestyle measures used to treat

296 patients living with diabetes. Imayama et al. (2011) performed a longitudinal study of 490

297 T1DM and 1,147 T2DM patients to investigate the determinants of health-related QoL (HRQoL).

298 The authors also found a higher HRQoL in patients with a high physical activity level.

299 Concerning TS, the results showed a relatively high score despite the negative impact of

300 diabetes on QoL, which has been reported in previous studies (Bradley \& Speight, 2002; Speight

301 \& Bradley, 2000; Speight et al., 2009). We could not detect any difference between the LADA

302 patients and the other groups in the DTSQ final score. Nevertheless, the hyperglycemia

303 frequency perception was worse in the insulin-treated LADA patients, although glycemic control

304 was not different between the groups. The increased hyperglycemia frequency perception in

305 the subjects with LADA may be attributable to previous poorer glycemic control compared with

306 type 1 diabetic patients under stable control. It is worth noting that the LADA subjects were

307 recruited from a specialized hospital clinic, where patients are usually referred from primary

308 care because of poor glycemic control. In contrast, the frequency of hypoglycemia perception

309 was similar between the LADA and T2DM patients and was clearly increased in T1DM patients.

310 This finding could be attributed to a higher intensity of insulin treatment in T1DM, which may

311 be associated with a higher frequency of hypoglycemia in these patients. Unfortunately, the

312 frequency of previous mild and severe hypoglycemia episodes was not assessed. Additionally, 
313

314

315

316

317

318

319

320

321

322

323

324

325

326

327

328

329

330

331

332

333

334

as expected from the exposure to insulin treatment, the insulin-treated LADA patients had an

increased frequency of hypoglycemia compared with the non-insulin treated T2DM patients.

Improved TS has been associated with optimal glycemic control (HbA1c $\leq 7 \%)$ in T2DM without

concomitant complications or insulin treatment (Biderman, Noff, Harris, Friedman, \& Levy,

2009). These findings are in line with those reported here. Furthermore, we found that physical

activity showed a positive association with TS, as in our previous study that involved the

subgroup of T2DM patients included in our study (Alcubierre et al., 2014); however, we could

not identify other studies addressing this specific issue in the diabetic population.

The current study has several limitations. A causal relationship between QoL, TS and related

factors could not be established because of the cross-sectional study design; however, the

variation in the QoL among patients was shown to be strongly influenced by the characteristics

that do not vary over time (Alva, Gray, Mihaylova, \& Clarke, 2014). The low number of patients

with LADA that were included is an important limitation. Therefore, the low number of subjects

in the LADA group raises a point of caution regarding the external validity of the current results.

Additionally, a significant number of potential subjects from the local LADA cohort refused to

participate. However, there were no differences between the participating LADA patients and

the non-participating LADA patients in the proportion of comorbidities that could affect the

main study outcomes. Thus, the current study should be considered an exploratory

investigation that raises awareness of the need for further studies of patient-oriented

outcomes in subjects with LADA. Additionally, the current study compared the QoL and TS of

LADA patients with that of patients with the two main classical types of diabetes. Another

limitation arises from the potential selection bias of the current study hospital setting. Subjects 
335 with worse glycemic control are referred to specialized care for further diagnostic work-up that

336 leads to the final diagnosis of LADA, while subjects with LADA who have better glycemic control

337 are likely to remain unidentified at the primary care level. Therefore, the current results may

338 not be extrapolated to the whole population of LADA subjects. The issue of poor QoL is very

339 relevant to patients with LADA who are ultimately referred to specialized care because of

340 unstable glycemic control and the need for insulin treatment. Finally, mental well-being may

341 have an impact on the main outcomes evaluated in the current study. Although mood items are

342 included in the ADDQoL-19 questionnaire, a proper evaluation of emotional or mental well-

343 being was not performed in this study. This latter issue should be taken into consideration in

344 future studies of patients with LADA.

345

346 CONCLUSIONS

347 In conclusion, in the current study we found that that LADA patients with DR who were

348 undergoing insulin treatment had a negative QoL compared with T2DM and T1DM patients.

349 Furthermore, the LADA patients undergoing insulin treatment perceived a greater frequency of

350 hyperglycemia than the other diabetic groups. Further research is warranted to study the status

351 and changes over time in the QoL and TS of patients with LADA in other settings and with a

352 larger number of patients. 


\section{REFERENCES}

354 Ahola, A. J., Saraheimo, M., Forsblom, C., Hietala, K., Sintonen, H., Groop, P.-H., \& FinDiane

355 Study, G. (2010). Health-related quality of life in patients with type 1 diabetes-association

356 with diabetic complications (the FinDiane Study). Nephrology Dialysis Transplantation, 25(6):1903-1908. http://doi.org/10.1093/ndt/gfp709

358

359

360

361

362

363

364

365

366

367

368

369

370

371

372

373

374

Alcubierre, N., Rubinat, E., Traveset, A., Martinez-Alonso, M., Hernandez, M., Jurjo, C., \& Mauricio, D. (2014). A prospective cross-sectional study on quality of life and treatment satisfaction in type 2 diabetic patients with retinopathy without other major late diabetic complications. Health and Quality of Life Outcomes, 12:131.

http://doi.org/10.1186/s12955-014-0131-2

Alva, M., Gray, A., Mihaylova, B., \& Clarke, P. (2014). The effect of diabetes complications of health-related quality of life: the importance of longitudinal data to address patient heterogeneity. Health Economics, 23:487-500.

Bernstein, M. S., Morabia, A., \& Sloutskis, D. (1999). Definition and prevalence of sedentarism in an urban population. American Journal of Public Health, 89(6):862-867. http://doi.org/10.2105/AJPH.89.6.862

Biderman, A., Noff, E., Harris, S. B., Friedman, N., \& Levy, A. (2009). Treatment satisfaction of diabetic patients: What are the contributing factors? Family Practice, 26(2):102-108. http://doi.org/10.1093/fampra/cmp007

Bradley, C. (1994). Diabetes Treatment Satisfaction Questionnaire (DTSQ). In Handbook of Psychology and Diabetes: A Guide to Psychological Measurement in Diabetes Research and Practice (pp. 111-112). 
375 Bradley, C., De Pablos-Velasco, P., Parhofer, K. G., Eschwge, E., Gönder-Frederick, L., \& Simon,

376 D. (2011). PANORAMA: A European study to evaluate quality of life and treatment

377 satisfaction in patients with type 2 diabetes mellitus - Study design. Primary Care Diabetes, 5(4):231-239. http://doi.org/10.1016/j.pcd.2011.04.004

379

380

381

382

383

384

385

386

387

388

389

390

391

392

393

394

395

396

Bradley, C., \& Speight, J. (2002). Patient perceptions of diabetes and diabetes therapy: assessing quality of life. Diabetes/metabolism Research and Reviews, 18 (Suppl 3):S64-9. http://doi.org/10.1002/dmrr.279

Bradley C, Todd C, Gorton T, Symonds E, Martin A, P. R. (1999). The development of an individualised questionnaire measure of perceived impact of diabetes on quality of life: the ADDQoL. Qual Life Research, 8:79-81.

Cabrera de León, A., Rodríguez-Pérez, M. D. C., Rodríguez-Benjumeda, L. M., Anía-Lafuente, B., Brito-Díaz, B., Muros de Fuentes, M., ... Aguirre-Jaime, A. (2007). Sedentary lifestyle: physical activity duration versus percentage of energy expenditure. Revista Española de Cardiologia, 60(3):244-250. http://doi.org/10.1016/S1885-5857(07)60148-0

Collins, M., O'Sullivan, T., Harkins, V., \& Perry, I. (2009). Quality of Life and Quality of Care in patients with diabetes experiencing different models of care. Diabetes Care, 32(4):603-5. http://doi.org/10.2337/dc08-1169.

Depablos-Velasco, P., Salguero-Chaves, E., Mata-Poyo, J., Derivas-Otero, B., Garcia-Sanchez, R., \& Viguera-Ester, P. (2014). Quality of life and satisfaction with treatment in subjects with type 2 diabetes: results in Spain of the PANORAMA study. Endocrinol Nutr, 61(1): 18-26. http://doi.org/10.1016/j.endonu.2013.05.005

Esteban y Peña, M. M., Hernandez Barrera, V., Fernández Cordero, X., Gil de Miguel, A., 
397

398

399

400

401

402

403

404

405

406

407

408

409

410

411

412

413

414

415

416

417

418

Rodríguez Pérez, M., Lopez-de Andres, a, \& Jiménez-García, R. (2010). Self-perception of health status, mental health and quality of life among adults with diabetes residing in a metropolitan area. Diabetes \& Metabolism, 36(4):305-11.

http://doi.org/10.1016/j.diabet.2010.02.003

Fourlanos, S., Dotta, F., Greenbaum, C. J., Palmer, J. P., Rolandsson, O., Colman, P. G., \& Harrison, L. C. (2005). Latent autoimmune diabetes in adults (LADA) should be less latent. Diabetologia, 48(11):2206-2212. http://doi.org/10.1007/s00125-005-1960-7

Gomis, R., Herrera-Pombo, J., Calderón, A., Rubio-Terrés, C., \& Sarasa, P. (2006). Validación del cuestionario "Diabetes treatment satisfaction questionnaire" (DTSQ) en la población española. Pharmacoeconomics, 3:7-20.

Hawa, M. I., Kolb, H., Schloot, N., Beyan, H., Paschou, S. A., Buzzetti, R., ... Leslie, R. D. (2013). Adult-onset autoimmune diabetes in Europe is prevalent with a broad clinical phenotype: Action LADA 7. Diabetes Care, 36(4):908-913. http://doi.org/10.2337/dc12-0931

Hawa, M. I., Thivolet, C., Mauricio, D., Alemanno, I., Cipponeri, E., Collier, D., ... Leslie, R. D. G. (2009). Metabolic syndrome and autoimmune diabetes: Action LADA 3. Diabetes Care, 32(1):160-164. http://doi.org/10.2337/dc08-1419

Hernandez, M., Mollo, A., Marsal, J. R., Esquerda, A., Capel, I., Puig-Domingo, M., ... Action LADA Consortium. (2015). Insulin secretion in patients with latent autoimmune diabetes (LADA): half way between type 1 and type 2 diabetes: action LADA 9. BMC Endocrine Disorders, 15(1):1. http://doi.org/10.1186/1472-6823-15-1

Hervás A, Zabaleta A, de Miguel G, Beldarrain O, D. J. (2002). Health related quality of life in patients with diabetes mellitus type 2. Diabetes Care, 25:464-470. 
419 Imayama, I., Plotnikoff, R. C., Courneya, K. S., \& Johnson, J. A. (2011). Determinants of quality of 420 life in adults with type 1 and type 2 diabetes. Health and Quality of Life Outcomes,

421

422

423

424

425

426

427

428

429

430

431

432

433

434

435

436

437

438

439

440

9(1):115. http://doi.org/10.1186/1477-7525-9-115

Isomaa, B., Almgren, P., Henricsson, M., Taskinen, M. R., Tuomi, T., Groop, L., \& Sarelin, L. (1999). Chronic complications in patients with slowly progressing autoimmune type 1 diabetes (LADA). Diabetes Care, 22(8):1347-1353.

http://doi.org/10.2337/diacare.22.8.1347

Leslie, R.D., Kolb, H., Schloot, N. C., Buzzetti, R., Mauricio, D., De Leiva, A., Yderstraede, K., Sarti, C., \& Thivolet, C. (2008). Diabetes classification: grey zones, sound and smoke: action LADA 1. Diabetes Metabolism Research and Reviews, 24(7):511-519. http://doi:10.1002/dmrr.877

Leslie, R. D., Palmer, J., Schloot, N. C., \& Lernmark, A. (2016). Diabetes at the crossroads: relevance of disease classification to pathophysiology and treatment. Diabetologia, 59(1):13-20. http://doi.org/10.1007/s00125-015-3789-z

Mancera-Romero, J., Carramiñana-Barrera, F., Muñoz-González, L., Guillén-Álvarez, P., MurilloGarcía, D., \& Sánchez-Pérez, M. R. (2016). Satisfaction of patients with type 2 diabetes mellitus after starting treatment with insulin. Semergen - Sociedad Española de Medicina Rural y Generalista, 42(5):298-306. http://doi.org/10.1016/j.semerg.2015.06.002

Mollo, A., Hernandez, M., Marsal, J. R., Esquerda, A., Rius, F., Blanco-Vaca, F., ... Mauricio, D. (2013). Latent autoimmune diabetes in adults is perched between type 1 and type 2: Evidence from adults in one region of Spain. Diabetes Metabolism Research and Reviews, 29(6):446-451. http://doi.org/10.1002/dmrr.2411 
441 Nicolucci, A., Cucinotta, D., Squatrito, S., Lapolla, A., Musacchio, N., Leotta, S., ... Coronel, G. 442 (2009). Clinical and socio-economic correlates of quality of life and treatment satisfaction 443 in patients with type 2 diabetes. Nutrition, Metabolism, and Cardiovascular Diseases,19(1):45-53. http://doi.org/10.1016/j.numecd.2007.12.005

Oliva, J., Fernandez-Bolanos, A., \& Hidalgo, A. (2012). Health- related quality of life in diabetic people with different vascular risk. BMC Public Health, 12(1):812. http://doi.org/10.1186/1471-2458-12-812

Shim, Y. T., Lee, J., Toh, M. P. H. S., Tang, W. E., \& Ko, Y. (2012). Health-related quality of life and glycaemic control in patients with Type 2 diabetes mellitus in Singapore. Diabetic Medicine: A Journal of the British Diabetic Association, 29(8): e241-8. http://doi.org/10.1111/j.1464-5491.2012.03689.x

Speight, J., \& Bradley, C. (2000). ADDQoL indicates negative impact of diabetes on quality of life 453 despite high levels of satisfaction with treatment. Diabetologia, 43 (Suppl 1): A225.

Speight, J., Reaney, M. D., \& Barnard, K. D. (2009). Not all roads lead to Rome-a review of 455 quality of life measurement in adults with diabetes. Diabetic Medicine: A Journal of the British Diabetic Association, 26(4):315-27. http://doi.org/10.1111/j.14645491.2009.02682.x

Stenstrom, G., Gottsater, A., Bakhtadze, E., Berger, B., \& Sundkvist, G. (2005). Latent Autoimmune Diabetes in Adults: Definition, Prevalence, Cell Function, and Treatment.

Sundaram, M., Kavookjian, J., Patrick, J. H., Miller, L.-A., Madhavan, S. S., \& Scott, V. G. (2007). 462 Quality of life, health status and clinical outcomes in Type 2 diabetes patients. Quality of 
463

464

465

466

467

468

469
Life Research: An International Journal of Quality of Life Aspects of Treatment, Care and Rehabilitation, 16(2):165-77. http://doi.org/10.1007/s11136-006-9105-0

Villar-López J, Lizán-Tudela L, Soto-Alvarez J, P.-M. S. (2009). Treatment satisfaction. Atención Primaria, 41(11):637-45.

Wilson, M., Moore, M. P., \& Lunt, H. (2004). Treatment satisfaction after commencement of insulin in Type 2 diabetes. Diabetes Res Clin Pract, 66(3):263-267.

http://doi.org/10.1016/j.diabres.2004.04.01 
